\title{
Caracterización de cepas de Klebsiella pneumoniae productora de $\beta$-lactamasa de espectro extendido aisladas en dos unidades de cuidados intensivos
}

\author{
Ana C. González, Beatriz Nieves, Marisé Solórzano, Jhon Cruz, Juan Puig y Magaly Moreno
}

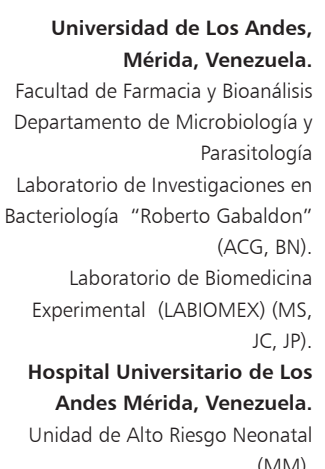

(MM).

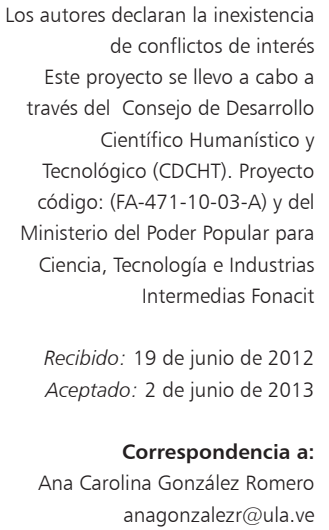

\section{Characterization of extended-spectrum $\beta$-lactamases-producing Klebsiella pneumoniae isolates of two intensive care units}

Background: Extended-spectrum $\beta$-lactamase (ESBL)- producing Klebsiella pneumonia is more the frequently implied bacterium infections nosocomiales in the Neonatal High Risk Unit (NHRU), and adult intensive care unit (aICU) in the The Andes University Hospital Autonomy Institute. Aim: To determine the microbiological and molecular characteristics associated with infections caused by this bacterium. Material and Methods: 17 strains of $K$. pneumoniae isolated from neonates from NHRU with nosocomial infections and 11 isolates from patients hospitalized in the aICU between June to November 2009 were characterized by in vitro agar diffusion test and MIC, the production of $\beta$-lactamase, the presence of bla $_{T E M}, b l a_{S H V} y$ bla ${ }_{C T X-M}$ genes and genotyping. Results: A high percentage of resistance to $\beta$-lactams and the presence of ES $\beta$ L TEM, SHV and CTX-M in 41.2 y $82 \%$ of strains obtained at HRNU and at aICU respectively were detected. BLEE $(+)$ phenotype was detected in $17,6 \%$ of HRNU strains and $91 \%$ of aICU strains. Genotypic analysis by REP-PCR detected several clones suggesting that resistant clones of ESBL-producing $K$. pneumoniae are endemic not only specific circulation and transmission of a single common among patients. Conclusion: $K$. pneumoniae strains that produce b-lactamases circulate in these critical units studied representing a therapeutic challenge that is necessary to fight against.

Key words: Nosocomial infection, Klebsiella pneumoniae, extended- spectrum $\beta$ - lactamase.

Palabras clave: Infección nosocomial, Klebsiella pneumoniae, $\beta$-lactamasa de espectro extendido.

\section{Introducción}

$K$ lebsiella pneumoniae es un patógeno bacteriano importante, asociado a infecciones en la comunidad y nosocomiales, especialmente en pacientes inmunocomprometidos. Las cepas de $K$. pneumoniae tienen el potencial para causar morbilidad y mortalidad, particularmente en las unidades de cuidados intensivos pediátricos y servicios quirúrgicos ${ }^{1}$.

Las $\beta$-lactamasas de espectro extendido ( $\beta \mathrm{LEE}$ ) son enzimas producidas por bacilos gramnegativos capaces de hidrolizar las cefalosporinas de amplio espectro y los monobactámicos, pero no las cefamicinas ni los carbapenémicos. Son $\beta$-lactamasas mediadas generalmente por plásmidos y derivan de otras enzimas con menor espectro hidrolítico. La aparición de las $\beta$ LEE ha dificultado enormemente el tratamiento de numerosas infecciones bacterianas porque estas cepas presentan, además de resistencia a la gran mayoría de los $\beta$-lactámicos, altas tasas de resistencia a antimicrobianos de otras familias ${ }^{2}$

La mayoría de los genes que codifican $\beta$ LEE son transmitidos por plásmidos y a menudo se encuentran en los transposones y los integrones, facilitando su movili- zación con otro determinante de resistencia. Por lo tanto, los genes que codifican $\beta$ LEE pueden ser fácilmente transferidos horizontalmente entre e intra especies ${ }^{3,4}$. Las $\beta$ LEE más frecuentes se han incluido en tres grupos: TEM, SHV y CTX-M. La enzima SHV se denomina sulfidrilo variable y se asocia con $K$. pneumoniae. La enzima TEM fue nombradas del paciente del que procedía, Temoneira, y fue descubierta por primera vez en Escherichia coli en Grecia. La $\beta$ LEE tipo CTX-M está relacionada con la $\beta$-lactamasa cromosomal de Kluyvera ascorbata ${ }^{5}$, la cual se reportó en 1989 y se caracterizó por hidrolizar mejor a cefalosporinas como cefuroxima, cefotaxima y cefepima que ceftazidima ${ }^{6}$. Este tipo de $\beta$ LEE se ha expandido rápidamente y es ahora uno de los tipos de $\beta$ LEE más dominantes en muchos países ${ }^{7,8}$.

En el medio nosocomial, las $\beta$ LEE son consideradas como causas importantes del incremento en la morbilidad y mortalidad de pacientes hospitalizados, de la prolongada estancia hospitalaria y del aumento de los costos globales de salud ${ }^{1,9}$.

Numerosos estudios señalan que las tasas de infección por $K$. pneumoniae productora de $\beta$ LEE causantes de brotes en las Unidades de Cuidados Intensivos (UCI) 
están en aumento. Debido al papel que ha jugado este microorganismo en estas unidades en el Instituto Autónomo Hospital Universitario de los Andes (IAHULA), se planteó un estudio con el propósito de determinar las características microbiológicas y moleculares asociadas a las infecciones nosocomiales causadas por cepas de K. pneumoniae aisladas de la Unidad de Alto Riesgo Neonatal (UARN) y la Unidad de Cuidados Intensivos Adultos (UCIa), contribuyendo de esta manera con la prevención y control de las infecciones.

\section{Materiales y Métodos}

Cepas bacterianas. El estudio se realizó con cepas de $K$. pneumoniae aisladas de pacientes hospitalizados en las unidades clínicas UARN y UCIa, durante el período comprendido entre junio y noviembre de 2009. El procesamiento de las muestras y el estudio microbiológico se llevó a cabo en el Laboratorio de Bacteriología Anaeróbica "Dr. Roberto Gabaldón", del Departamento de Microbiología y Parasitología de la Facultad de Farmacia; y el estudio molecular en el Laboratorio de Biología y Medicina Experimental (LABIOMEX), Universidad de Los Andes.

El estudio fue aprobado por las instancias del IAHULA involucradas, y no requirió procedimientos clínicos distintos a los realizados habitualmente en la atención de los pacientes.

\section{Estudio microbiológico}

- Identificación y biotipificación. Las cepas de K. pneumoniae se identificaron mediante los procedimientos descritos por Koneman y cols. ${ }^{10}$. La biotipificación de las cepas se realizó mediante el sistema comercial API 20E (BioMerieux, Francia). El procedimiento se realizó siguiendo las instrucciones de la casa fabricante.

\section{- Prueba de susceptibilidad antimicrobiana}

Método de difusión del disco. A las cepas de K. pneumoniae se les determinó la susceptibilidad a agentes antimicrobianos mediante la prueba de difusión del disco en agar (Kirby-Bauer) de acuerdo a las instrucciones del CLSI ${ }^{11}$. Se ensayaron los siguientes agentes antimicrobianos:

- $\beta$-lactámicos: cefotaxima (30 $\mu \mathrm{g}$ bioDiscs), ceftazidima (30 $\mu \mathrm{g}$ bioDiscs), cefoxitina (30 $\mu \mathrm{g} \mathrm{BBL),} \mathrm{cefepime}$ (30 $\mu \mathrm{g} \mathrm{BBL).}$

- Combinaciones de inhibidores de $\beta$-lactamasas: ampicilina/sulbactam (10/10 $\mu \mathrm{g}$ Difco); amoxicilina/ác. clavulánico (10/10 $\mu \mathrm{g}$ Difco); piperacilina/tazobactam (10/10 $\mu \mathrm{g}$ Difco).

- Monobactámicos: aztreonam (30 $\mu \mathrm{g}$ Difco).

- Carbapenémicos: imipenem (10 $\mu$ g HIMEDIA).

- Quinolonas: ciprofloxacina (5 $\mu$ g HIMEDIA).
- Aminoglucósidos: gentamicina (10 $\mu \mathrm{g}$ Difco), amikacina $(30 \mu \mathrm{g}$ Difco).

Se utilizó como cepa control una cepa de Escherichia coli ATCC 25922.

- Determinación de la concentración inhibitoria mínima (CIM). Se empleó el método Etest ${ }^{\circledR}$ (BioMérieux, Francia), siguiendo las instrucciones de la casa comercial. Los antimicrobianos que se utilizaron en estos ensayos fueron los siguientes: ceftazidima, piperacilina/tazobactam, gentamicina, amikacina y aztreonam. Los resultados se interpretaron siguiendo las instrucciones de la casa comercial y del CLSI ${ }^{11}$.

- Detección fenotípica de $\beta$-lactamasas de espectro extendido ( $\beta \mathrm{LEE}$ ) se realizó mediante el método descrito por Pitout y cols. ${ }^{12}$.

\section{Estudio molecular}

Detección de genes bla ${ }_{T E M}$ bla ${ }_{S H V}$ y bla ${ }_{C T X-M}$ en cepas de K. pneumoniae mediante reacción de polimerasa en cadena-RPC. La extracción de ADN se realizó por calentamiento a $90^{\circ} \mathrm{C}$. La secuencia del iniciador TEM1 fue (TCCGCTCATGAGACAATAACC) y TEM2 (TTGGTCTGACAGTTACCAATGC). Tamaño del fragmento $750 \mathrm{pb}^{13}$.

La secuencia del iniciador SHV1 fue (TGGTTATGCGTTATATTCGCC) y SHV2 (GGTTAGCGTTGCCAGTGCT). Tamaño del fragmento $370 \mathrm{pb}^{13}$.

El iniciador CTX-M1 fue (5-TTTGCGATGTGCAGTACCAGTAA-3) y CTX-M2 (5- CGATATCGTTGGTGGTGCCATA-3) Tamaño del fragmento $540 \mathrm{pb}^{14}$.

Se incluyó un control negativo con una mezcla de todos los componentes de la mezcla de reacción, excepto el ADN, y como control positivo se utilizó una cepa de $K$. pneumoniae productora de $\beta$ LEE tipo TEM, SHV, CTX$\mathrm{M}$ caracterizada previamente y donada gentilmente por el centro de colección de microorganismo. Instituto de Biología Experimental. Universidad Central de Venezuela

Los programas de amplificación para TEM, SHV se realizaron bajo las condiciones descritas por Coque y cols. ${ }^{13}$ y para CTX-M por Edelstein y cols. ${ }^{14}$.

\section{Genotipificación}

Reacción en cadena de polimerasa de elementos palindrómicos extragénicos repetitivos (REP-PCR). La extracción de ADN cromosomal se realizó mediante el método descrito por Snelling y cols. ${ }^{15}$. Los oligonucleótidos utilizados se basaron en elementos palindrómicos extragénicos repetitivos de ADN altamente conservados, cuya secuencia es la siguiente:

REP-1 (5' - III ICGICGICATCIGGC-3').

REP-2 (5' - ICGICTTATCIGGCCTAC-3') (Invitrogen) Versalovic ${ }^{16}$. 
Con cada muestra se preparó la mezcla de reacción para la amplificación, la cual se desarrolló con un volumen final de $12,5 \mu 1$, con $0,08 \mathrm{U} / \mu 1$ de Taq polimerasa, $25 \mathrm{mM}$ de $\mathrm{Mgcl}_{2}, 0,2 \mathrm{mM}$ de cada dNTPs, 50 pmoles de cada uno de los oligonucleótidos opuestos y la muestra de ADN genómico a una dilución de 1/1.000.

Se incluyó un control negativo con una mezcla de todos los componentes anteriormente citados, excepto el ADN. La amplificación se realizó en un termociclador (Eppendorff), el cual fue programado de acuerdo a las especificaciones descritas por Salazar y cols. ${ }^{17}$.

\section{Criterios de interpretación}

El tamaño de los fragmentos generados por electroforesis de los productos de RPC se determinó por comparación con la escalera de peso molecular. La presencia o ausencia de bandas en una línea del gel se determinó por

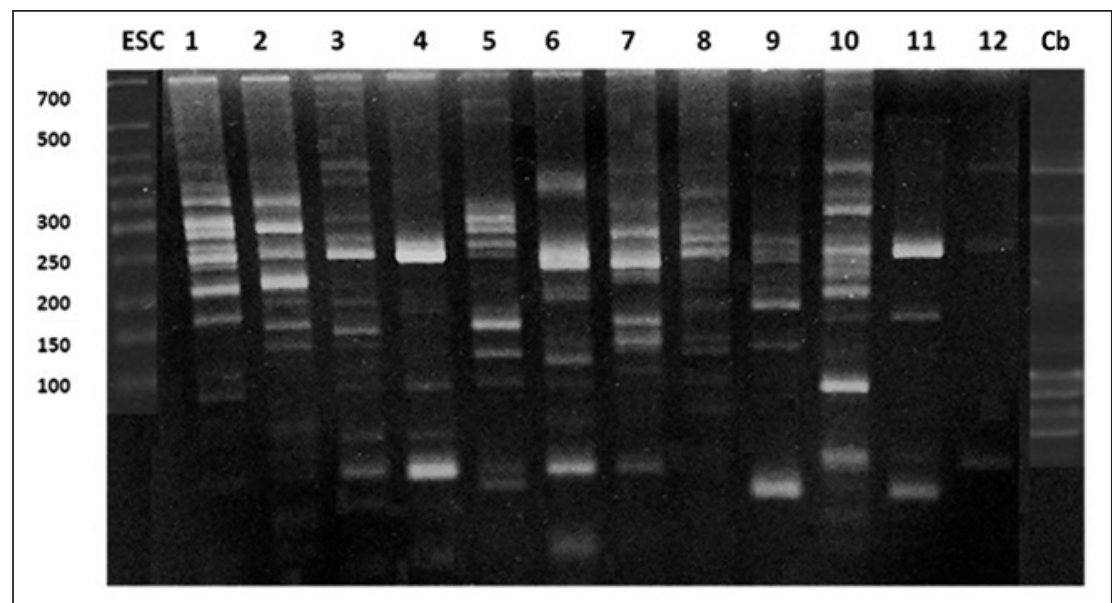

Figura 1(A). Patrón de bandas obtenidas por REP-PCR de las cepas de $K$. pneumoniae aisladas de la UARN del IAHULA. Esc: escalera, Línea 1 (Genotipo 1N), Línea 2 (Genotipo 2N), Línea 3 (Genotipo $3 N)$, Línea 4 (Genotipo 4N), Línea 5 (Genotipo 5N), Línea 6 (Genotipo 6N), Línea 7 (Genotipo 7N), Línea 8 (Genotipo 8N), Línea 9 (Genotipo 9N), Línea 10 (Genotipo 10N). Cb: Cepa de K. pneumoniae responsable del brote 2007 ocurrido en la unidad.

Figura 1 (B). Patrón de bandas obtenidas por REP-PCR de las cepas de K. pneumoniae aisladas de la UARN del IAHULA. Esc: escalera, Línea 11 (Genotipo 11N), Línea 12 (Genotipo 12N), Línea 13 (Genotipo 13N), Línea 14 (Genotipo 14N), Línea 15 (Genotipo 15N), Línea 16 (Genotipo 16N), Línea 17 (Genotipo 17N).Cb: Cepa de K. pneumoniae responsable del brote 2007 ocurrido en la unidad. inspección visual de los patrones, por tres observadores diferentes. Cepas con patrones de RPC iguales sobre las bases de similitud en cuanto al número y posición de todas las bandas mayores se consideraron del mismo genotipo. Pequeñas diferencias en la intensidad y forma de las bandas mayores o pérdida de bandas débiles fueron ignoradas (Tenover y cols. ${ }^{18}$ ).

\section{Resultados}

Se caracterizaron 17 cepas de $K$. pneumoniae aisladas de neonatos con infección nosocomial hospitalizados en la UARN, así como 11 cepas aisladas de pacientes hospitalizados en la UCIa, durante el período de estudio.

Las cepas de K. pneumoniae aisladas de los neonatos mostraron un alto porcentaje de resistencia a ampicilina (100\%), ampicilina/sulbactam (100\%), amoxicilina/ ác. clavulánico (100\%) seguido de amikacina (94\%), ceftazidima (94\%), cefotaxima (94\%), aztreonam (94\%), gentamicina (82\%) y una sensibilidad de $100 \%$ a imipenem, $94 \%$ a cefoxitina, $88 \%$ a ciprofloxacina y $58,8 \%$ a piperacilina/tazobactam.

En las cepas de $K$. pneumoniae aisladas de los pacientes hospitalizados en la UCIa, se observó similares porcentaje de resistencia a los $\beta$-lactámicos y su combinación con inhibidores que en las cepas aisladas de la UARN.

De las 17 cepas de $K$. pneumoniae aisladas de los neonatos, en siete $(41,2 \%)$ cepas se detectaron los tres genes bla (TEM, SHV y CTX-M) investigados; sin embargo, las siete eran del fenotipo $\beta$ LEE (-). Los valores de CIM de ceftazidima ante estas cepas fueron de $64 \mu \mathrm{g} / \mathrm{ml}$. El resto de las cepas sólo poseían los genes bla (TEM y SHV); no obstante, sólo tres $(3 / 17 ; 17,6 \%)$ eran del fenotipo $\beta$ LEE (+) (Tabla 1).

Todas las 17 cepas fueron genotípicamente diferentes mediante REP-PCR (Tabla 1, Figura 1A y 1B).

De las cepas aisladas en la UCIa, nueve (82\%) poseían los tres genes bla (TEM, SHV y CTX-M) investigados y, a diferencia de las cepas aisladas de la UARN, las nueve eran del fenotipo $\beta$ LEE $(+)$. El resto de las cepas (n: 2) sólo poseían los genes de TEM y SHV y una de ellas era del fenotipo $\beta$ LEE (-) (Tabla 1). En suma, 10/11 (91\%) de las cepas estudiadas en la UCIa eran BLEE $(+)$.

Se encontraron siete genotipos diferentes entre las cepas de $K$. pneumoniae aisladas de esta unidad, predominando el genotipo I (Tabla 1, Figura 2).

\section{Discusión}

Klebsiella pneumoniae es un patógeno de importancia en el ambiente hospitalario, frecuentemente relacionado con infecciones nosocomiales en las unidades de cuidados intensivos y salas de pediatría, donde se aíslan cada vez con mayor frecuencia cepas resistentes a cefalosporinas 
Tabla 1. Características de las cepas de $K$. pneumonie aisladas de los pacientes con infección nosocomial en las unidades de Alto Riesgo Neonatal y Cuidados intensivos de adultos del HULA

\begin{tabular}{|c|c|c|c|c|c|c|c|c|c|}
\hline $\begin{array}{l}\text { Cepa } n \text { de } \\
\text { laboratorio }\end{array}$ & Unidad & $\begin{array}{c}\text { CIM CAZ } \\
(\mu \mathrm{g} / \mathrm{ml})\end{array}$ & $\begin{array}{l}\text { CIM P/T } \\
(\mu \mathrm{g} / \mathrm{ml})\end{array}$ & $\begin{array}{l}\text { CIM AZT } \\
(\mu \mathrm{g} / \mathrm{ml})\end{array}$ & $\begin{array}{c}\text { CIM AMK } \\
(\mu \mathrm{g} / \mathrm{ml})\end{array}$ & $\begin{array}{c}\text { CIM G } \\
(\mu \mathrm{g} / \mathrm{ml})\end{array}$ & $\begin{array}{c}\text { Fenotipo de } \\
\beta L E E\end{array}$ & Tipo de $\beta$ LEE & Genotipo \\
\hline 1 & UARN & $64 R$ & $3 S$ & $128 \mathrm{R}$ & $192 R$ & $24 R$ & - & TEM,SHV,CTX & $1 \mathrm{~N}$ \\
\hline 2 & UARN & $64 R$ & $3 S$ & $96 \mathrm{R}$ & $192 R$ & $24 R$ & - & TEM,SHV & $2 \mathrm{~N}$ \\
\hline 3 & UARN & $96 \mathrm{R}$ & $2 S$ & $64 R$ & $128 \mathrm{R}$ & $2 S$ & - & TEM,SHV & $3 \mathrm{~N}$ \\
\hline 4 & UARN & $16 R$ & $3 S$ & $16 R$ & $192 R$ & $192 R$ & - & TEM,SHV & $4 N$ \\
\hline 5 & UARN & $128 \mathrm{R}$ & 35 & $128 \mathrm{R}$ & $192 R$ & $256 R$ & + & TEM,SHV & $5 \mathrm{~N}$ \\
\hline 6 & UARN & $64 \mathrm{R}$ & $16 \mathrm{~S}$ & $128 R$ & $256 R$ & $256 R$ & + & TEM,SHV & $6 \mathrm{~N}$ \\
\hline 7 & UARN & $64 \mathrm{R}$ & $16 \mathrm{~S}$ & $128 R$ & $256 R$ & $256 R$ & + & TEM,SHV & $7 \mathrm{~N}$ \\
\hline 8 & UARN & $16 R$ & $256 R$ & $16 R$ & $128 R$ & $128 \mathrm{R}$ & - & TEM,SHV & $8 \mathrm{~N}$ \\
\hline 9 & UARN & $0,50 \mathrm{~S}$ & $2 S$ & $0,64 \mathrm{~S}$ & $1,50 \mathrm{~S}$ & $6 S$ & - & TEM,SHV & $9 \mathrm{~N}$ \\
\hline 10 & UARN & $64 \mathrm{R}$ & $3 S$ & $96 R$ & $128 \mathrm{R}$ & $96 R$ & - & TEM,SHV,CTX & $10 \mathrm{~N}$ \\
\hline 11 & UARN & $64 \mathrm{R}$ & $192 R$ & $256 R$ & $256 R$ & $256 R$ & - & TEM,SHV,CTX & $11 \mathrm{~N}$ \\
\hline 12 & UARN & $64 R$ & $256 R$ & $16 R$ & $64 R$ & $128 \mathrm{R}$ & - & TEM,SHV,CTX & $12 \mathrm{~N}$ \\
\hline 13 & UARN & $64 \mathrm{R}$ & $256 R$ & $16 R$ & $256 R$ & $96 R$ & - & TEM,SHV,CTX & $13 N$ \\
\hline 14 & UARN & $96 R$ & $2 S$ & $32 R$ & $48 \mathrm{R}$ & $2 S$ & - & TEM,SHV & $14 \mathrm{~N}$ \\
\hline 15 & UARN & $64 R$ & $256 \mathrm{R}$ & $16 R$ & $128 R$ & $96 R$ & - & TEM,SHV,CTX & $15 N$ \\
\hline 16 & UARN & $16 R$ & $256 R$ & $16 R$ & $64 \mathrm{R}$ & $64 \mathrm{R}$ & - & TEM,SHV & $16 \mathrm{~N}$ \\
\hline 17 & UARN & $64 R$ & $256 R$ & $16 R$ & $64 R$ & $128 R$ & - & TEM,SHV,CTX & $17 \mathrm{~N}$ \\
\hline 1 & UCla & $48 \mathrm{R}$ & 325 & $192 R$ & $256 R$ & $256 R$ & + & TEM,SHV,CTX & $1 \mathrm{~A}$ \\
\hline 2 & UCla & $48 \mathrm{R}$ & 325 & $192 \mathrm{R}$ & $256 R$ & $256 R$ & + & TEM,SHV,CTX & $1 \mathrm{~A}$ \\
\hline 3 & UCla & $64 \mathrm{R}$ & $3 S$ & $64 \mathrm{R}$ & $256 R$ & $256 R$ & + & TEM,SHV,CTX & $2 \mathrm{~A}$ \\
\hline 4 & UCla & $16 R$ & $2 S$ & $24 R$ & 35 & $256 \mathrm{R}$ & + & TEM,SHV,CTX & $3 \mathrm{~A}$ \\
\hline 5 & UCla & $32 R$ & 485 & $128 R$ & $256 R$ & $256 R$ & + & TEM,SHV,CTX & $4 \mathrm{~A}$ \\
\hline 6 & UCla & $16 R$ & 125 & $32 \mathrm{R}$ & $16 R$ & $1.5 \mathrm{~S}$ & + & TEM,SHV,CTX & $5 \mathrm{~A}$ \\
\hline 7 & UCla & $32 R$ & 325 & $192 R$ & $16 R$ & $256 \mathrm{R}$ & - & TEM,SHV & $4 \mathrm{~A}$ \\
\hline 8 & UCla & $128 \mathrm{R}$ & $2 S$ & $192 R$ & $256 R$ & $192 R$ & + & TEM,SHV & $6 \mathrm{~A}$ \\
\hline 9 & UCla & $128 \mathrm{R}$ & $2 S$ & $192 R$ & $256 \mathrm{R}$ & $192 R$ & + & TEM,SHV,CTX & $7 \mathrm{~A}$ \\
\hline 10 & UCla & $128 R$ & $3 S$ & $256 R$ & $256 \mathrm{R}$ & $192 R$ & + & TEM,SHV,CTX & $7 \mathrm{~A}$ \\
\hline 11 & UCla & $64 R$ & $192 R$ & $256 R$ & $256 R$ & $256 R$ & + & TEM,SHV,CTX & $1 \mathrm{~A}$ \\
\hline
\end{tabular}

de tercera generación con resistencia combinada a múltiples antimicrobianos ${ }^{1,19}$. Debido a la gravedad de estas infecciones, la investigación constante y la aplicación de medidas de control reconocidas y efectivas, enmarcadas dentro de un programa de vigilancia, constituyen estrategias que pueden reducir sustancialmente la incidencia y prevalencia de las infecciones nosocomiales ${ }^{20}$.

Durante el desarrollo de este proyecto de infecciones nosocomiales, llevado a cabo en la UARN y UCIa del HULA, los bacilos gramnegativos representaron el mayor porcentaje de aislamiento en ambas unidades. En la UARN, $22 \%$ de los aislados pertenecían a la familia Enterobacteriacea, siendo $K$. pneumoniae la especie más frecuente $(53,1 \%)$. Por otro lado, en la UCIa, $K$. pneumoniae se aisló con una frecuencia relativa de 55\%

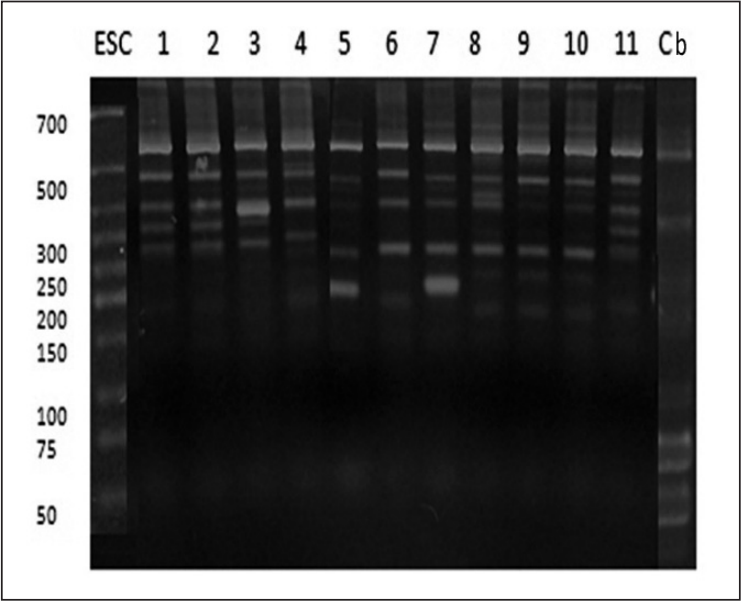

Figura 2. Patrón de bandas obtenidas por REP-PCR de las cepas de $K$. pneumoniae aisladas de la UCla del IAHULA. Esc: escalera, Línea 1, 2 y 11 (Genotipo 1A), Línea 3 (Genotipo 2A), Línea 4 (Genotipo 3A), Línea 5 y 7 (Genotipo 4A), Línea 6 (Genotipo 5A), Línea 8 (Genotipo 6A), Línea 9 y 10 (Genotipo 7A), Cb :Cepa de K. pneumoniae responsable del brote 2007 ocurrido en la UARN, C-: Control negativo. 
dentro del grupo de bacilos gramnegativos fermentadores. Estos resultados son similares a los encontrados en otras unidades de cuidados especiales en países de América Latina, donde aproximadamente $60 \%$ de las infecciones son causadas por bacilos gramnegativos, entre ellos, $K$. pneumoniae $^{21}$. En investigaciones anteriores realizadas en la UARN del HULA, esta bacteria ha sido causa de brotes y se ha aislado de la sangre de los neonatos, de las soluciones parenterales y de las soluciones jabonosas, así como también, de las manos del personal ${ }^{1,19}$. A pesar de las medidas de control y prevención de las infecciones nosocomiales implementadas en la UARN, la tasa de aislamiento de K. pneumoniae sigue siendo alta.

La resistencia a los agentes antimicrobianos en bacilos gramnegativos causantes de infecciones nosocomiales se ha convertido en un hallazgo común en los grandes hospitales en todo el mundo ${ }^{22}$. En la actualidad, una de las mayores preocupaciones de la comunidad científica internacional es la creciente incidencia de infecciones producidas por $K$. pneumoniae resistente a múltiples agentes antimicrobianos, especialmente a los $\beta$-lactámicos ${ }^{20}$. Este fenómeno fue igualmente observado en el presente estudio. En la UARN, 87\% de las cepas fueron resistentes a ocho o más agentes antimicrobianos, especialmente a los $\beta$-lactámicos y a los aminoglucósidos. De igual manera, en la UCIa se observó un alto porcentaje de resistencia a estos dos grupos de antimicrobianos.

Con la proliferación de cepas multi-resistentes, el problema de las infecciones intrahospitalarias causadas por $K$. pneumoniae es aún mayor, ya que dan lugar a incrementos de las tasas de mortalidad, de las estancias hospitalarias y de los costos de atención ${ }^{4}$. El alto nivel de mortalidad a consecuencia de infecciones asociadas a la atención de salud con cepas de $K$. pneumoniae multi-resistente es alarmante; parece ser que la carencia de control de las fuentes de contaminación e higiene ha causado la diseminación de los microorganismos entre los pacientes, probablemente debido a que $K$. pneumoniae $\beta$ LEE $(+)$ coloniza transitoriamente las manos del personal de salud del hospital, facilitando su transmisión ${ }^{1}$

De aquí que es importante diferenciar cepas individuales y caracterizar los mecanismos de resistencia en este tipo de microorganismos ${ }^{4}$. El principal problema de resistencia entre los patógenos aislados en las unidades de cuidados especiales del HULA, es la prevalencia de $K$. pneumoniae productora de $\beta$ LEE, quizás como consecuencia de la exposición incrementada a las cefalosporinas de amplio espectro ampliamente utilizadas en estas unidades en la terapia empírica inicial, aunado al hecho de que $50 \%$ de los neonatos de quienes se aisló K. pneumoniae multi-resistente eran prematuros, de bajo peso al nacer y sometidos a algún procedimiento invasor, al igual que los pacientes infectados en la UCIa, donde $100 \%$ estaban sometidos a procedimientos invasores.
Es importante resaltar que los antimicrobianos carbapenémicos aún presentan actividad contra las cepas de $K$. pneumoniae productoras de $\beta$ LEE aisladas en estas unidades del HULA, a diferencia de los estudios de resistencia realizados por el CIDEIM en Colombia, donde ya existen cepas de $K$. pneumoniae productoras de metalo- $\beta$ lactamasas que le confieren resistencia a carbapenémicos, circulando en varios hospitales del país ${ }^{24}$. No obstante, su uso debe vigilarse permanentemente, por cuanto su utilización indiscriminada podría originar la selección de cepas de bacilos gramnegativos resistentes a carbapenémicos. Las cepas aisladas en la UARN mantienen la susceptibilidad en $100 \%$, a carbapenémicos, cefamicinas y quinolonas. Mientras que las cepas aisladas en la UCIa sólo permanecieron sensibles a carbapenémicos y cefamicinas pero no a quinolonas.

La prueba fenotípica de producción de $\beta$ LEE permitió detectar que $82 \%$ de las cepas aisladas de la UCIa eran productoras de $\beta$ LEE. Mientras que un bajo porcentaje $(17,6 \%)$ de las cepas aisladas de la UARN eran fenotipo $\beta$ LEE $(+)$, aunque a través de RPC se demostró que las restantes negativas eran portadoras de los genes bla. Ello puede deberse a la presencia de enzimas que actúan contra inhibidores de $\beta$-lactámicos, hiperproducción de enzimas, asociación con enzimas tipo AmpC o a la presencia de otros tipos de mecanismos de resistencia que interfieren con la prueba ${ }^{4}$.

Los resultados de esta investigación revelan la presencia de $\beta$ LEE tipo TEM, SHV y CTX-M en las dos unidades estudiadas y se demostró que algunas cepas eran portadoras de más de un gen $b l a_{\text {(TEM, SHV y СTX-M) }}$, lo cual es similar a los hallazgos reportados por otros investigadores $^{4,25}$. Las enzimas más comúnmente encontradas en Latinoamérica son SHV-2, SHV-4, SHV-5, CTX-M-2, CTX-M-8 y PER-2 ${ }^{9,26,27}$. En el presente trabajo, aunque la mayoría de las $\beta$ LEE eran del tipo TEM y SHV, las CTX-M se encontraron en mayor proporción en la UCIa (82\%) (9/11). En Colombia, las enzimas CTX-M, especialmente las del grupo 1 , se están reportando con mayor frecuencia ${ }^{28,4}$, mientras que en estudios realizados en Brasil predominan las CTX-M-2 en K. pneumoniae asociadas a brotes ${ }^{21}$.

Es importante señalar que al realizar el análisis genotípico mediante REP-PCR se detectaron varios clones en ambas unidades y al analizarlas en conjunto no coincidieron los patrones de bandas, lo cual sugiere que los clones que circulan en la UARN son diferentes a los que circulan en la UCIa. Incluso, al comparar los genotipos aislados en la UARN durante este estudio, con los obtenidos en un estudio realizado en el 2007 por González y cols. ${ }^{1}$, en esa misma unidad, se pudo observar que se trata de genotipos distintos. La mayoría de las cepas mostró una amplia diversidad, lo cual sugiere que existen cepas resistentes específicas no endémicas más que la circulación y trans- 
misión de un aislado habitual entre pacientes. Los genes que codifican las $\beta$ LEE, probablemente sean diseminados vía la transferencia de un determinante de resistencia entre los aislados clínicos. De hecho, en un estudio preliminar que se lleva a cabo actualmente con el propósito de verificar la presencia de plásmidos conjugativos, y si además éstos portaban los determinantes de resistencia en las cepas de K. pneumoniae, se encontró que 17 de estas cepas poseían determinantes de resistencia transferibles.

El ensayo de REP-PCR es útil como una alternativa para la tipificación rápida; sin embargo, es conveniente confirmar los resultados con procedimientos que tengan mayor poder de discriminación, como la electroforesis en gel de campo pulsado ${ }^{1,22}$.

Agradecimientos. Al Fondo Nacional de Ciencia, Tecnología e Innovación (FONACIT). Proyecto código: 2012001234 y al Consejo de Desarrollo Científico Humanístico y Tecnológico (CDCHT). Proyecto código: FA-471-10-03-A por la financiación del estudio. De igual manera, a los pacientes y al personal de las unidades de cuidados intensivos neonatal y de adultos del IAHULA.

\section{Resumen}

Introducción: Klebsiella pneumoniae productora de $\beta$-lactamasa de espectro extendido ( $\beta \mathrm{LEE}$ ) es la bacteria más frecuentemente implicada en infecciones nosocomiales en las Unidades de Alto Riesgo Neonatal (UARN) y Cuidados intensivos de adulto (UCIa) del Instituto Autónomo Hospital Universitario de Los Andes. Objetivo: Determinar las características microbiológicas y moleculares asociadas a las infecciones causadas por esta bacteria. Material y Métodos: Se caracterizaron la susceptibilidad in vitro por difusión en agar y CIM, la presencia de $\beta$-lactamasa y la presencia de genes $b l a_{T E M}, b l a_{S H V} \mathrm{y}$ bla $_{\text {CTX-M }}$ y la genotipificación en 17 cepas de $K$. pneumoniae aisladas de neonatos con infección nosocomial, así como 11 aisladas de pacientes hospitalizados en la UCIa entre junio y noviembre de 2009. Resultados: Esta investigación reveló un alto porcentaje de resistencia a $\beta$-lactámicos y la presencia de genes tipo bla ${ }_{T E M}, b l a_{S H V}$ y $b l a_{C T X-M}$ en 41,2 y $82 \%$ de las cepas procedentes de UARN y UCIa, respectivamente. Producción de BLEE se detectó en $17,6 \%$ cepa de UARN y en $91 \%$ de las pertenecientes a UCIa. Mediante el análisis genotípico REP-PCR se detectaron varias clones lo cual sugiere que existen clones resistentes de K. pneumoniae productora de $\beta$ LEE específicas no endémicas más que la circulación y transmisión de un aislado habitual entre pacientes. Conclusión: En las unidades críticas estudiadas circulan cepas de $K$. pneumoniae productoras de $\beta$-lactamasas constituyendo un problema terapéutico importante y necesario de combatir.

\section{Referencias bibliográficas}

1.- González A, Gil F, Solórzano M, Cruz J, Puig J, Suárez M, Nieves B. Brote por Klebsiella pneumoniae multirresistente y productora de $\beta$-lactamasa de espectro extendido en una unidad de alto riesgo neonatal. Rev Chilena Infectol 2011; 28 (1): 28-34.

2.- Díaz M, Hernández J, Martínez-Martínez L, Rodríguez-Bano J, Pascual A y Grupo de Estudio de Infección Hospitalaria (GEIH) Escherichia coli y Klebsiella pneumoniae productoras de betalactamasas de espectro extendido en hospitales españoles: segundo estudio multicéntrico (proyecto GEIH-BLEE 2006). Enferm Infecc Microbiol Clin 2009; 27(9): 503-10.

3.- Eckert C, Gautier V, Arlet G. DNA sequence analysis of the genetic environment of various blaCTX-M genes. J Antimicrob Chemother 2006; 57: 14-23.

4.- Gaitán S, Espinal P. y Grupo de Investigación en Resistencia Bacteriana, Región Caribe. Caracterización molecular de Escherichia coli y Klebsiella pneumoniae productores de $\beta$-lactamasas de espectro extendido en hospitales de la Región Caribe, Colombia. Rev Chilena Infectol 2009; 26 (3): 239-46.
5.- Humeniuk C, Arlet G, Gautier V, Grimont P, Labia R, Philippon A. $\beta$-lactamases of Kluyvera ascorbata, probable progenitors of some plasmid-encoded CTX-M types. Antimicrob Agents Chemother 2002; 46: 3045-9.

6.- Lin C F, Hsu S K, Chen C H, Huang J R, Lo H H. Genotypic detection and molecular epidemiology of extended-spectrum $\beta$-lactamase-producing Escherichia coli and Klebsiella pneumoniae in a regional hospital in central Taiwan. J Med Microbiol 2010; 59: 665-71.

7.- Lee S G, Jeong S H, Lee H, Kim C K, Lee Y, Koh E, et al. Spread of CTX-M-type extendedspectrum betalactamases among bloodstream isolates of Escherichia coli and Klebsiella pneumoniae from a Korean hospital. Diagn Microbiol Infect Dis 2009; 63: 76-80.

8.- Livermore D, Canton R, Gniadkowski M, Nordmann P, Rossolini G, Arlet G, et al. CTX-M: changing the face of ESBLs in Europe. J Antimicrob Chemother 2007; 59: 165-74.

9.- Minarini L A, Climaco E C, Guimaraes D B, Ferreira J C, Palazzo I C, Martínez R, et al. Clonal transmission of ESBL-producing Klebsiella spp. at a university hospital in Brazil. Curr Microbiol 2008; 56 (6): 587-91.

10.- Koneman E, Allen S, Janda W, Schreckenberger
P, Winn W. Diagnóstico Microbiológico. Texto y Atlas a Color. 6ta Edición. Editorial Médica Panamericana. Buenos Aires, Argentina. 2007. Págs. 44-335.

11.- Clinical and Laboratory Standards Institute. Performance Standards for Antimicrobial Susceptibility Testing; 19th Informational Supplement. CLSI M100-S19.2010. Clinical and Laboratory Standards Institute, Wayne, PA.

12.- Pitout J, Reisbig M, Venter E, Church D, Hanson M. Modification of the double-disk test for detection of Enterobacteriaceae producing extended-spectrum and AmpC $\beta$-lactamases. J Clin Microbiol 2003; 41 (8): 3933-5.

13.- Coque T M, Oliver A, Pérez-Díaz J C, Baquero F, Cantón R. Genes encoding TEM-4, SHV-2, and CTX-M-10 extended-spectrum $\beta$-lactamases are carried by multiple Klebsiella pneumoniae clones in a single hospital (Madrid, 1989 to 2000). Antimicrob Agents Chemother 2002; 46: 500-10.

14.- Edelstein M, Pimkin M, Palagin I, Edelstein I, Stratchounski L. Prevalence and molecular epidemiology of CTX-M extended-spectrum $\beta$-lactamase-producing Escherichia coli and Klebsiella pneumoniaein Russian hospitals. Antimicrob Agents Chemother 2003; 47: 3724-32. 
15.- Snelling A, Gerner P, Hawkey P, Heritage J, Parnel P, Poster C. Validation of use of whole-cell repetitive extragenic palindromic secuence based PCR (REP-PCR) for typing strains belonging to the Acinetobacter calcoaceticus-Acinetobacter baumannii complex and application of the method to the investigation of a hospital outbreak. J Clin Microbiol 1996; 34 (5): 1193-202.

16.- Versalovic J, Koeuth T, Lupsky J. Distribuction of repetitive DNA sequences in Eubacteria and application to fingerprinting of bacterial genome. Nucleic Acids Res 1991; 19 : 6823-31.

17.- Salazar E Z, Nieves B, Araque M, Velazco E, Ruiz J, Vila J. Outbreak of infection with Acinetobacter strain RUH 1139 in an intensive care unit. Infect Control Hosp Epidemiol 2005; 27: 397-403.

18.- Tenover F, Arbeit R, Goering R, Mickelsen P, Murray B, Persing D, et al. Interpreting chromosomal DNA restriction patterns produced by pulsed-field gel electrophoresis: criteria for bacterial strain typing. J Clin Microbiol 1995; 33 (9): 2233-9.

19.- Calderas Z, Nieves B, Araque M, Torres A, Mosqueda, N. Klebsiella pneumoniae productora de $\beta$ LEE como agente etiológico de septicemia en una unidad de cuidados intensivos. Resumen de las XXVI Jornadas Venezolanas de Microbiología Dr. José Esparza "Infecciones Emergentes". Valencia, noviembre 1999; p. 33.

20.- Christian N, Roye-Green K, Smikle M. Molecular epidemiology of multidrug resistant extended spectrum beta-lactamase producing Klebsiella pneumoniae at a Jamaican hospital, 2000-2004. BMC Microbiol 2010; 10: 27.

21.- de Oliveira García D, Doi Y, Szabo D, Adams-Haduch J M, Vaz T M, Leite D, et al. Multiclonal outbreak of Klebsiella pneumoniae producing extended-spectrum $\beta$-lactamase CTX-M-2 and novel variant CTX-M-59 in a neonatal intensive care unit in Brazil. Antimicrob Agents Chemother 2008; 54(5): 1790-3.

22.- García A, García E, Hernández A, Ruiz J, Yagüe G, Herrero J, Gómez J. Bacteriemias por Escherichia coli productora de betalactamasas de espectro extendido (BLEE): significación clínica y perspectivas actuales. Rev Esp Quimioter 2011; 24 (2): 57-66.

23.- Cirz R, Romesberg F. Induction and inhibition of ciprofloxacin resistance-conferring mutations in hypermutator bacteria. Antimicrob Agents Chemother 2006; 50 (1): 220-5.

24.- López Vargas J, Echeverri Toro Lina. $K$. pneumoniae: ¿la nueva "superbacteria"?
Patogenicidad, epidemiología y mecanismos de resistencia. IATREIA 2010; 23 (2): 25-30.

25.- Smith E, Geun S, Thomson K, Larone D, Hanson N. Letters to Editor. Klebsiella pneumoniae isolate producing at least eight different $\beta$-lactamases, including AmpC and KPC $\beta$-lactamases. Antimicrob Agents Chemother 2007; 51 (2): 800-1.

26.- Casellas J, Nannini E, Radice M, Cocconi E, Lejona S, Noemí Borda N, et al. Estudio de un brote debido a aislados de Klebsiella pneumoniae productores de betalactamasas de espectro extendido en un centro asistencial de Rosario-Argentina. Rev Panam Infectol 2005; 7 (4): 21-7.

27.- Vignoli R, Cordeiro N, Seija V, Schelotto F, Radice M, Ayala J, et al. Entorno genético de CTX-M-2 en aislamientos de Klebsiella pneumoniae provenientes de pacientes hospitalizados en Uruguay. Rev Argent Microbiol 2006; 38: 84-8.

28.- Mantilla J, Reguero M, González E, García I, Leal A, Espinal P, et al. Caracterización molecular de un brote por Klebsiella pneumoniae productora de CTX-M-12 en la unidad de cuidado intensivo neonatal de un hospital colombiano. Biomédica 2006; 26 : 408-14. 